\title{
The Study of Kinetic Energy of Ion and Sheath Thickness in Magnetized Plasma Sheath
}

\author{
Roshan Chalise ${ }^{*}$ and Raju Khanal \\ Central Department of Physics, Tribhuvan University, Kirtipur - 3, Kathmandu, Nepal
}

\begin{abstract}
In all plasma applications, sheath formed in front of a material wall is responsible for the flow of particles and energy towards the wall. The kinetic energy of ion and sheath thickness in magnetized plasma sheath region has been numerically investigated by using a kinetic trajectory simulation model for different magnetic field and its obliqueness. It has been revealed that, the kinetic energy of ions reaching the material wall can be controlled by the strength of applied magnetic field and its orientation. Kinetic energy of ion increases when we move towards the wall, whereas the kinetic energy of electrons decreases as expected, which becomes prominent as the strength and obliqueness of the field increase. It is found that by increasing the magnetic field strength, there is an increase in the ion energy and a decrease in the sheath thickness.
\end{abstract}

Key words: Plasma, magnetized plasma sheath, presheath.

\section{Introduction}

The study of magnetized plasma sheath is one of the important research problems in plasma experiments, so the correlative works both of experiment and theory are developed greatly during the past several years [1-4], and significant number of work have been done in recent time [5-8] by using different models. Calculation of the ion flux from plasma to a surface of a cathode is one of the key problems of a theory of a plasma-surface interaction. For example, without knowing the ion flux, one cannot estimate the energy brought by the ions to the surface, also one cannot find the characteristics of the near-cathode space-charge sheath created by the ions; and in particular, the strength of the electric field at the cathode surface cannot be estimated, therefore, the electron emission current density from the surface into the plasma also remains indeterminate [9]. PIII (Plasma immersion ion implantation) is a relatively new technology that is used to modify material surface properties. In PIII process, a negatively biased target

Corresponding author: Roshan Chalise, M.Sc, research fields: magnetized plasma sheath and plasma sheath simulation. E-mail:plasma.roshan@gmail.com. is placed in the plasma. The resulting electric field is concentrated in the target vicinity, which is a sheath where quasi-neutrality breaks down. This electric field accelerates ions to the high energy towards the target. High energy ions are implanted to the surface and modify the surface properties for a wide range of materials. Since the main ion acceleration is concentrated in the sheath, the sheath behavior is extremely important in PIII. It is clear that keeping the sheath thickness in the optimal range is an important problem. One of the ways to control the sheath thickness may be the use of a magnetic field. Hence, the sheath study is vital for many application of the plasma e.g. material processing, plasma sputtering, plasma etching and for confinement of the plasma in fusion devices. Energetic ion bombardment is an essential part of plasma etching and deposition processes. Etch rates, etch profile deposition rates, damage and deposited film properties are all depended on the ion flux and kinetic energy of the ions. To obtain the acceptable results, ion flux and the energy must be carefully optimized and controlled [10]. Plasma particles and heat flux reaching the material walls depend on the characteristics of the plasma sheath 
located between the plasma and the material surface. Recently, it has become significantly important for diverter physics in magnetic fusion devices. There are several key factors which determine the energy transmission through the sheath [11]. An interaction between the plasma and a solid wall, and the formation of a boundary layer around it are almost as old as plasma physics itself. The problem of magnetized plasma boundary has great importance in material processing by the plasma and in confining fusion plasma in magnetic fusion devices. The importance of the plasma boundary layer in an external magnetic field has drawn much attention in recent years. Nonetheless, the physics of the plasma boundary is still not perfectly understood [12]. For all practical applications of plasma, it is necessary to know the kinetic energy of the ions for the energy transmission, hence the problem is still a recent issue.

In this work, we have numerically studied the kinetic energy of ion and electron, potential and ion flux in magnetized plasma-sheath region that formed adjacent to an absorbing wall with presheath plasma on the other side. We have used the KTS (Kinetic Trajectory Simulation) model [13] to obtain solution to a non-neutral, time independent, collisionless plasma sheath and hence calculated kinetic energy of ion and electron for different magnitude and obliqueness of the magnetic field.

\section{Basic about KTS}

KTS is an iterative method for numerically calculating self-consistent, time independent kinetic plasma states in some given bounded spatial regions. The characteristic of KTS is that the distribution function of particle species involved are directly calculated by solving the related kinetic equations along the respective collisionless particle trajectory. In order to obtain the distribution function at any point $(\vec{x}, \vec{v})$ of the phase-space, we trace the related trajectories of phase-space where the distribution function is known. Here we assume the electrons and ions velocity distribution function at the sheath edge to be cut-off Maxwellian in such a way that the most important requirement of the presheath-sheath transition are satisfied, i.e. quasineutrality, the sheath-edge singularity condition, continuity of the first three moments of each species, and the kinetic Bohm criterion [13].

In the general case of time-dependent, collisional kinetic theory, the species-s velocity distribution function describes the Boltzmann equation:

$$
\frac{d f(\vec{x}, \vec{v}, t)}{d t}=\left[\frac{\partial}{\partial t}+\vec{v} \cdot \frac{\partial}{\partial \vec{x}}+\vec{a}^{s} \cdot \frac{\partial}{\partial \vec{v}}\right] f(\vec{x}, \vec{v}, t)=C^{s}
$$

with $\vec{a}^{s}(\vec{x}, \vec{v}, t)=\frac{q^{s}}{m^{s}}[\vec{E}(\vec{x}, t)+\vec{v} \times \vec{B}(\vec{x}, t)]$

where, $\vec{E}(\vec{x}, t)$ and $\vec{B}(\vec{x}, t)$ are the macroscopic (i.e. locally averaged) electromagnetic fields, $\vec{a}^{s}$ is the macroscopic acceleration of species-s particles (i.e. it's acceleration in these fields), and $C^{s}$ is the species-s collision term. The kinetic Boltzmann Eq. (1) for collisionless cases takes the well known from Vlasov Eq:

$$
\left(\frac{\partial}{\partial t}+\vec{v} \cdot \frac{\partial}{\partial \vec{x}}+\vec{a}^{s} \cdot \frac{\partial}{\partial \vec{v}}\right) f(\vec{x}, \vec{v}, t)=0
$$

i.e., $f(\vec{x}, \vec{v}, t)=$ constant. This means that the velocity distribution function is constant for an observer moving along a collisionless trajectory. Hence, the distribution function at every point along the trajectory can be obtained if it's value at one point (i.e., at the boundary) is known.

\section{Magnetized Plasma Sheath Model}

In the present work, we restrict ourselves to time independent, collisionless, and electromagnetic problems, as it is appropriate for the sheath regions. The $1 d 3 v$ model of magnetized plasma sheath is shown schematically in Fig. 1.

The region of interest or simulation region is bounded by two parallel planes situated at $x=0$ and $x=L$ and the plasma consists of only electrons and 


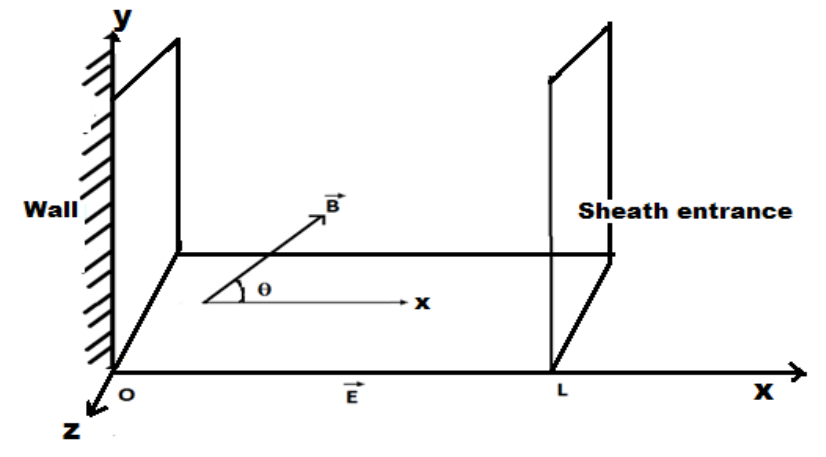

Fig. 1 Schematic diagram of the plasma sheath model.

singly charged ions. We have specified $x=L$ as the "sheath entrance" which separates the non-neutral, collisionless sheath region $(x<L)$ from the quasineutral collisional presheath region $(x>L)$. Similarly, an absorbing wall is specified by $x=0$. The magnetic field lies in the $x-y$ plane such that

$$
\vec{B}=B_{0}[\cos \theta \hat{x}+\sin \theta \hat{y}]
$$

We assume that the plasma particles (electrons and ions) enter the simulation region from the sheath entrance with cut off Maxwellian velocity distribution functions, the wall doesn't emit any particles and both boundaries are perfectly absorbing. Accordingly, the electron velocity distribution function is given by,

$$
f^{e}(x, v)=A^{e} \exp \left[-\left(\frac{v_{x}^{2}+v_{y}^{2}+v_{z}^{2}}{v_{v f}^{e}}\right)+\frac{e \phi(x)}{k T^{e}}\right] \Theta\left[v_{c}^{e}(x)-v_{x}\right]
$$

where, $v_{c}^{e}(x)=\sqrt{\frac{2 e\left[\phi(x)-\phi_{0}\right]}{m^{e}}}$ is the electron cut off velocity at $x, \mathrm{k}$ is the Boltzmann constant and $\Theta(\mathrm{x})$ is the Heaviside step function, i.e.,

$$
\begin{array}{cc}
\Theta(\mathrm{x})=1 & \text { if } \mathrm{x} \geq 0 \\
=0 & \text { otherwise }
\end{array}
$$

The ion velocity distribution function at $x=L$ is given by

$$
f^{i}(L, v)=A^{i} \exp \left[-\left(\frac{\left(v_{x}-v_{m}^{i}\right)^{2}+v_{y}{ }^{2}+v_{z}{ }^{2}}{v_{t f}^{i}}\right)\right] \Theta\left(v_{c L}^{i}-v_{x}\right)
$$

where, $v_{t f}^{s}=\sqrt{\frac{2 k T^{s}}{m^{s}}}$ is the species-s (ion and electron) thermal velocity, $v_{m L}^{i}$ is the ion "Maxwellian-maximum" velocity at $x=L$ and $v_{c L}^{i}$ $\left(v_{c L}^{i}<0\right)$ is the ion cut off velocity at $x=L$. In the core plasma, the particle distribution would obviously be Maxwellian; however, in case of sheath formation, the ions are acclerated towards the wall so that they become shifted Maxwellian as given by Eq. (6). In addition, for the Bohm criterion to be satisfied by the ions, they must have attained certain minimum velocity $\left(v_{c L}^{i}\right)$ at the sheath entrance. As the electrons are retracted and reflected by the negative potential wall, their distribution gets cut-off at the sheath entrance as given by Eq. (4).

The starting parameters for our simulation are the kinetic parameters given at the sheath entrance, which can also be calculated by giving presheath parameters at the sheath boundary. In case of given presheath parameters, we solve a set of presheath-sheath transition equation, whose solution yield all necessary kinetic sheath parameters at the sheath entrance as well as the potential at the wall. This method of coupling presheath-sheath transition equation has been termed as the "Coupling Scheme" [13]. The potential at $x=L$ is always chosen equal to zero and we restrict ourselves to potential distribution $\phi(x)$, which decrease monotonically from $\phi=0$ at $x=L$ to $\phi_{w}<0$ at $x=0$ obtained from the coupling scheme. Once all the starting parameters at the sheath entrance are known, we solve the ion kinetic equations, for different discretized ion injection velocities up to the wall. This gives the ion velocities and their corresponding distribution function in the entire sheath region which on integration yields ion density distribution. Average kinetic energy of ions can be obtained by:

$$
E^{i}=\frac{1}{2} \frac{m^{i}}{n_{x}^{i}} \int_{-\infty-\infty}^{\infty} \int_{-\infty-\infty}^{\infty}\left(v_{x}^{2}+v_{y}^{2}+v_{z}^{2}\right) f_{x}^{i} d v_{x} d v_{y} d v_{z}
$$

Electron density distribution, on the other hand, is calculated analytically using

$$
n^{e}(x)=A^{e} \int_{-\infty}^{+\infty} d v_{x}^{+\infty} \int_{-\infty}^{+\infty} d v_{y}^{+\infty} \int_{-\infty}^{+\infty} d v_{z}\left[-\left(\frac{v_{x}^{2}+v_{y}^{2}+v_{z}^{2}}{v_{t f}^{e^{2}}}\right)\right] \Theta\left(v_{c x}^{e}-v_{x}\right)
$$

which yield density in terms of potential as

$$
n^{e}(\phi)=n_{L}^{e} \exp \left[\frac{e \phi(x)}{k T_{i}^{e}}\right]\left[\frac{1+e r f \sqrt{\frac{e\left(\phi(x)-\phi_{0}\right)}{k T_{f}^{e}}}}{1+e r f \sqrt{\frac{-e \phi_{0}}{k T_{f}^{e}}}}\right]
$$


Thus, the obtained ion and electron densities are used in Poisson equation, which results in new potential profile. The calculation is then iterated unless the final self-consistent state is reached.

Average kinetic energy of electron can be calculated analytically using

$$
E_{x}^{e}=\frac{1}{2} \frac{m^{e}}{n_{x}^{e}} \int_{-\infty}^{\infty} \int_{-\infty}^{\infty} \int_{-\infty}^{\infty}\left(v_{x}^{2}+v_{y}^{2}+v_{z}^{2}\right) f_{x}^{e} d v_{x} d v_{y} d v_{z}
$$

This yields the kinetic energy in terms of potential

$$
E^{e}={ }_{2}^{1} m^{e} v_{t f}^{e^{2}}\left\{\frac{3}{2}-\frac{\sqrt{\frac{e\left(\phi(x)-\phi_{0}\right)}{k T_{f}^{e}}} \exp \left[-\left(\frac{e\left(\phi(x)-\phi_{0}\right)}{k T_{f}^{e}}\right)\right]}{\sqrt{\pi}\left(1+e r f \sqrt{\frac{e\left(\phi(x)-\phi_{0}\right)}{k T_{f}^{e}}}\right)}\right\}
$$

\section{Results and Discussion}

Figs. 2 and 3 show the self consistent kinetic energy of ions and electrons versus normalized distance from the wall for a given magnetic field perpendicular and parallel to the wall.

From the above plot we see that the presence of an oblique magnetic field strongly affects the ion energy in a collisionless magnetized sheath. The kinetic energy of the ions at the sheath entrance is minimum, and increases inside the sheath region and attains its maximum values at the wall. This is attributed to the fact that the ions are accelerated by the negative wall potential and gains more and more energy when moving closer to the wall.

In the case of perpendicular magnetic field at $0^{\circ}$, the particles experience only the electric field, but in the case of $90^{\circ}$, the charged particles experience maximum magnetic force and hence gyrated by magnetic field as well as accelerated by electric field. For a constant magnetic field, ion kinetic energy at the wall increases with increased angle of applied field as shown in Fig. 4.

Fig. 5 shows the self-consistent kinetic energy profile of ion for different values of magnetic field at fixed $\theta\left(45^{\circ}\right)$. As discussed earlier, the kinetic energy of ions at the sheath entrance is minimum and increases inside the sheath region and attains its maximum values at the wall. As we increase the magnetic field, ions feel more magnetic forces, hence, ion density decreases such that the kinetic energy of ion increases and ion flux also increases. Increase in ion kinetic energy at the wall with the increase in magnetic field is shown in Fig. 6.

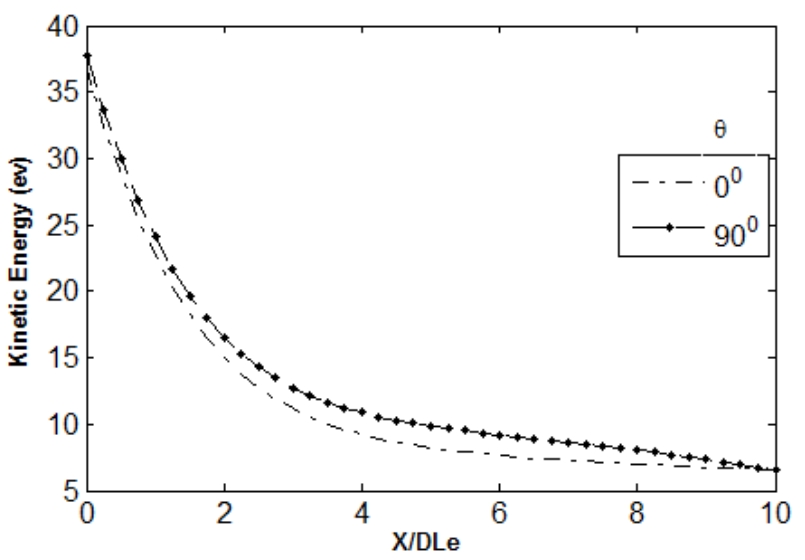

Fig. 2 Kinetic energy of ion for a given magnetic field (150 $\mathrm{mT})$ at $0^{\circ}$ and $90^{\circ}$ with the wall.

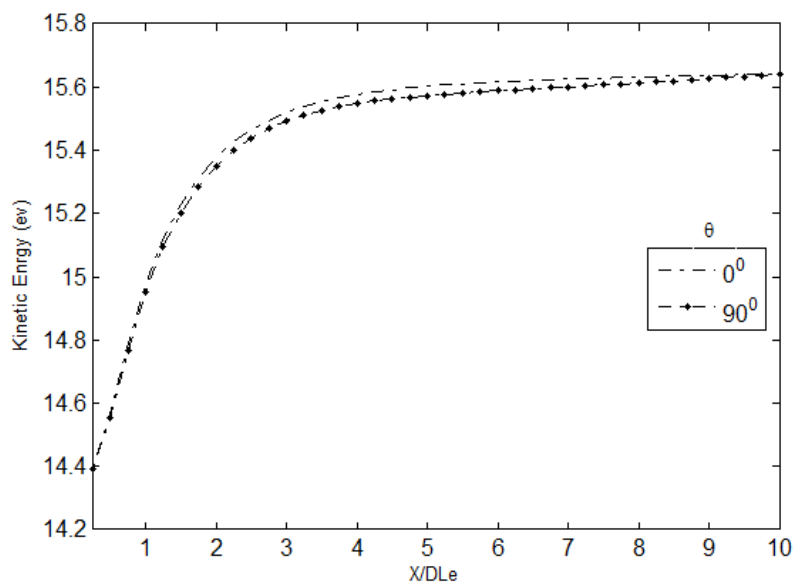

Fig. 3 Kinetic energy of electron for a given magnetic field $(150 \mathrm{mT})$ at $0^{\circ}$ and $90^{\circ}$ with the wall.

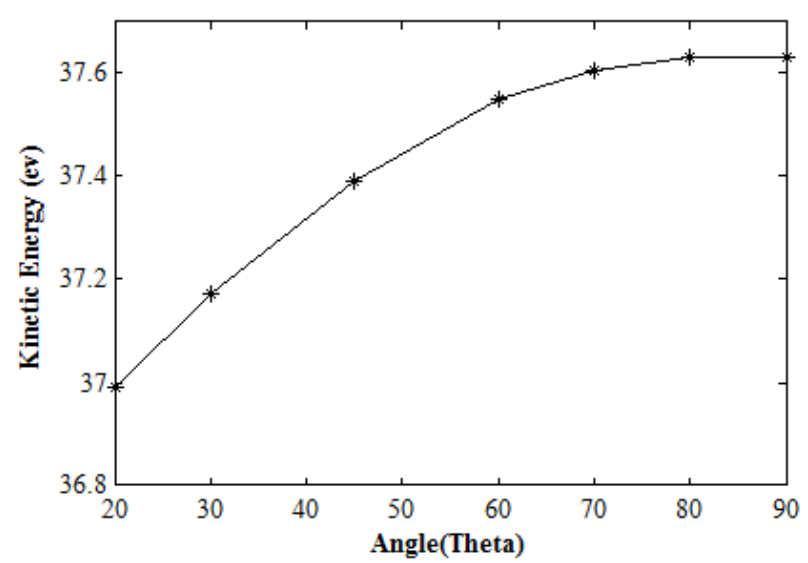

Fig. 4 Kinetic energy of ion at the wall for a given magnetic field $(150 \mathrm{mT})$ at different angles $(\theta)$. 


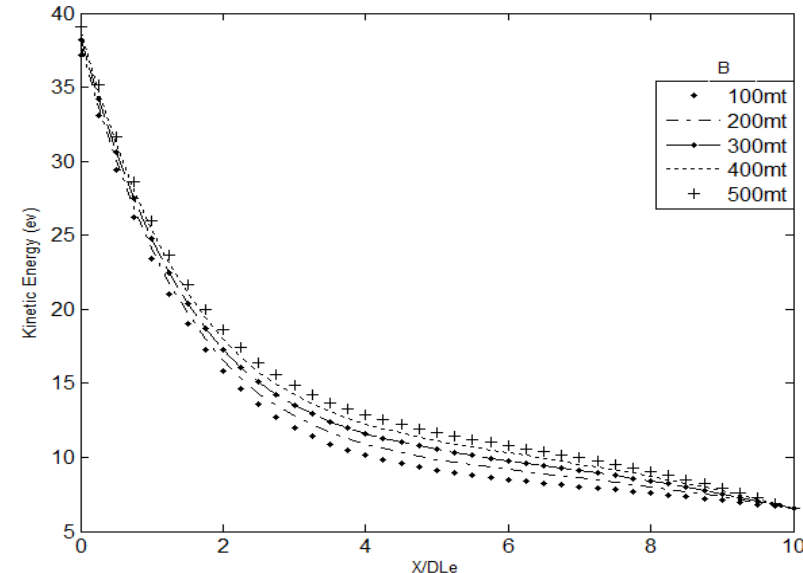

Fig. 5 Kinetic energy of ion for different value of magnetic field at fixed $\theta\left(45^{\circ}\right)$.

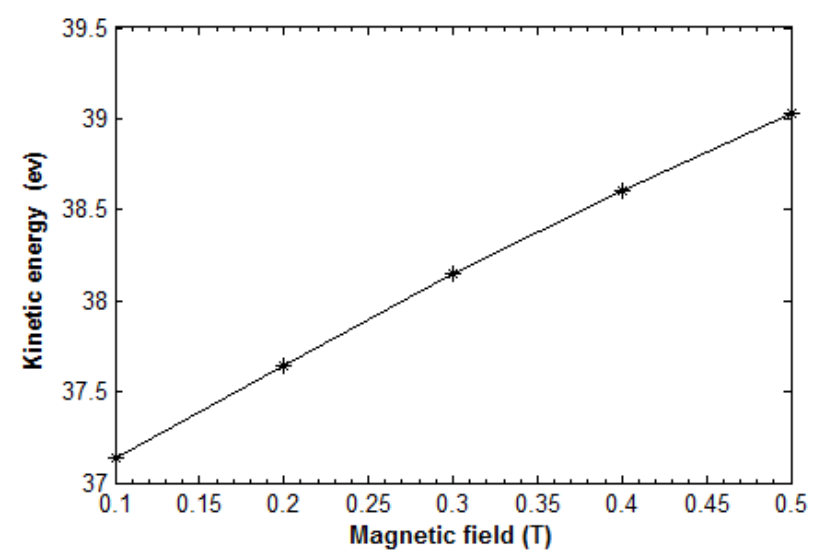

Fig. 6 Kinetic energy of ion at the wall for different magnetic field at a fixed angle $\theta\left(45^{\circ}\right)$.

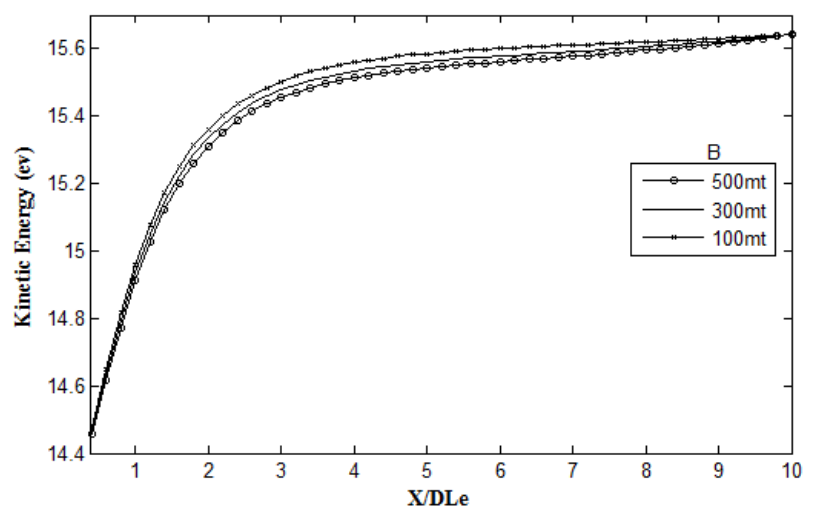

Fig. 7 Kinetic energy of electrons for different value of magnetic field at fixed $\theta\left(45^{\circ}\right)$.

Fig. 7 shows the self-consistent kinetic energy profile of electron for different values of magnetic fields at fixed $\theta\left(45^{\circ}\right)$. From the plot we see that kinetic energy of electron is maximum at the sheath entrance, then decreases gradually and acquired its

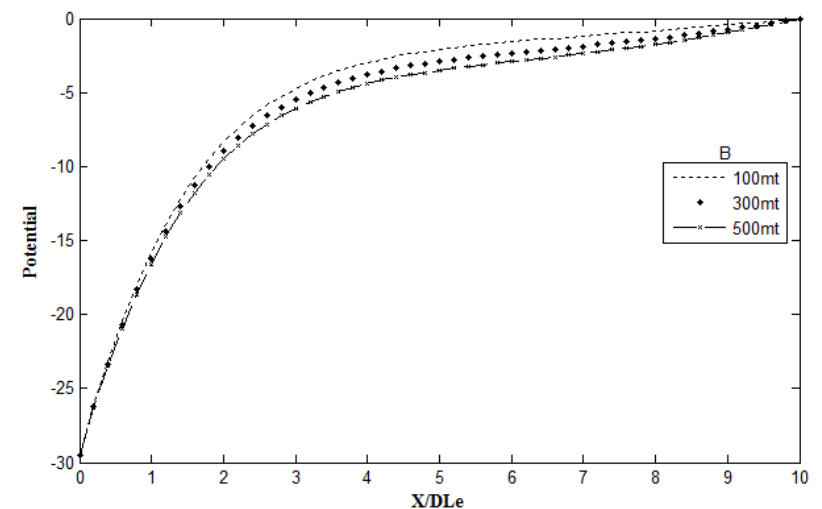

Fig. 8 Electric potential profile for different value of magnetic field at fixed $\theta\left(45^{0}\right)$.

minimum value at the wall. The decrease in the energy is due to the repulsive negative potential in the sheath region. As we increase the magnetic field, electrons experience increased magnetic force, hence the energy is also decreased. The energy at the wall is constant due to the fact that potential is same for all cases. This is also in consistent with the fact that the magnetic field is dominant only towards the presheath boundary, whereas the electric field prevails close to the wall [1].

Fig. 8 shows the self consistent sheath potential. With the increase of applied magnetic field, the sharply bending point of potential curve moves towards the wall, so that widths between this bending curve point and wall decrease more and more while increasing magnetic field. This means sheath width (thickness) decrease when the value of applied magnetic field increased.

\section{Conclusions}

It has been found that the magnetic field plays an important role in the plasma sheath region. The most prominent effect is ion kinetic energy, both for varying angle as well as magnitude of the field. For electrons, the effect is not so prominent, which is due to the fact that ions are dominant in the sheath. This result implies that the particle energy profile in the sheath region can be altered by varying the strength of magnetic field or its orientation. When increasing magnetic field, ion energy at the wall also increases, which enhances the flux of ion reaching the wall. It is 
also observed that ion energy reaching the wall increases as the obliqueness increased. It is found that by increasing the strength of the oblique magnetic field, sheath thickness decreases but flux of ion increase. The result plays an important role in the proper understanding and control of magnetized plasma sheath region. The present results may find applications in plasma processing; for example, in ion implantation, sputtering, material surface modification and semiconductor surface engineering etc.

\section{References}

[1] Chodura, R. 1982. "Plasma-Wall Transition in an Oblique Magnetic Field.” Phys. Fluids 25: 1628.

[2] Riemann, K. U. 1994. "Theory of the Plasma-sheath Transition in an Oblique Magnetic Field." Contributions to Plasma Physics 34: 127.

[3] Ahedo, E. 1997. "Structure of the Plasma-Wall Interaction in an Oblique Magnetic Field.” Phys. Plasmas. 4: 4419.

[4] Tskhakaya, D., and Kuhn, S. 2005. "The Magnetised Plasma-Wall Transition: Theory and PIC Simulation.” Plasma Phys. Control. Fusion 47A: 327.

[5] Masoudi, S. F., Esmaeili, S S., and Jazavandi, S. 2009. "Ion Dynamics in Plasma Sheath under the Effect of E
X B and Collisional Forces.” Vaccum 84: 382-6.

[6] Havlickova, E., and Hrach, R. 2010. "Numerical investigations of sheath structure in presence of magnetic field." Journal of Physics: Conference Series 207: 012030.

[7] Chalise, R., and Khanal, R. 2012. "A Kinetic Trajectory Simulation Model for Magnetized Plasma Sheath.” Plasma Phys. Control Fusion 54: 095006.

[8] Robertson, S. 2013. "Sheaths in Laboratory and Space Plasmas. (Topical Review) Plasma.” Plasma Phys. Controlled Fusion 55: 093001.

[9] Benilov, M.S. 1995. "The Ion Flux from a Thermal Plasma to a Surface.” J. Phys. D: Appl. Phys. 28: 286.

[10] Sobolewski, M. A. 2004. "Monitoring Sheath Voltages and Ion Energies in High-Density Plasmas Using Noninvasive Radio-Frequency Current and Voltage Measurements.” J. Appl. Phys. 95: 4593.

[11] Takamura, S., Ye, M. Y., Kuwabara, T., and Ohno, N. 1998. "Heat Flows through Plasma Sheaths.” Phys. of Plasma 5: 2151.

[12] horamabadi, M .K., Ghomi, H., and Shukla, P. K. 2011. "Numerical Investigation of the Ion Temperature Effects on Magnetized DC Plasma Sheath.” Journal of Applied Physics 109: 073307.

[13] Khanal, R. 2002. “A Kinetic Trajectory Simulation Model for Bounded Plasmas.” PhD Thesis, University of Insbruck. 